\title{
COSMIC STRING SPACETIME IN DILATON GRAVITY AND FLAT ROTATION CURVES
}

\author{
Tae Hoon Lee* \\ Department of Physics, Soongsil University, Seoul 156-743 Korea
}

(Dated: January 1, 2019)

\begin{abstract}
In dilaton gravity theories, we consider a string-like topological defect formed during $U(1)$ gauge symmetry-breaking phase transition in the early Universe, and far from the cosmic string we have vacuum solutions of the generalized Einstein equation. We discuss how they can be related to the flatness of galactic rotation curves.
\end{abstract}

PACS numbers: $04.50 .+\mathrm{h}, 11.27 .+\mathrm{d}, 95.35 .+\mathrm{d}$

\section{INTRODUCTION}

Topological defects of various kinds could be generated when symmetry-breaking phase transitions took place in the early Universe. Defects such as global monopoles or cosmic strings were thought of as the seeds for galaxy and large-scale structure formation,[1] and their remnants might remain as galactic dark matter.

The observed flatness[2] of spiral galaxy rotation curves suggests the existence of dark matter whose energy density goes like $1 / r^{2}$. Since the global monopole which was found by Barriola and Vilenkin[3] as a solution of Einstein's equations has energy density proportional to $1 / r^{2}$, it was suggested by Nucamendi and others[4] that the global monopole could account for some fraction of the galactic dark matter. Even though Harari and Loustó[5] noticed that the monopole core mass is negative and that there are no bound orbits, Nucamendi et al. [4] showed that there is an attractive region where bound orbits exist, by the introduction of a nonminimal coupling of gravity to the global monopole. Various, generalized scalar fields coupled to gravity with minimal coupling $[6]$ and with nonminimal coupling[7] were also studied in Einstein's theory as candidate dark matter. On the other hand, Banerjee et al. [4] noted that the global monopole in Brans-Dicke theory also exerts gravitational pull on a test particle moving in its spacetime. Recently we thus have studied in more detail the global monopole in Brans-Dicke theoy and discussed the importance of the $\ln r$ term of a gravitational potential in its possible relation to flat rotation curves.[8] Such ln $r$ terms can appear in various other cases[9] including global cosmic strings.[10]

The cosmic string is another solution of Einstein's equations in the effectively (2+1)-dimensional spacetime. It was found about 20 years ago and has been extensively studied[10] because of its possibly important role in the large-scale structure formation.[1] Since the energy density generated by long global strings varies[10] as $1 / r^{2}$ as global monopoles and it thus was thought to be responsible for the flatness of rotation curves in quasars,[11] we suggest it would be related to the flatness of rotation curves in spiral galaxies also. Even if a gauge cosmic string induces only a deficit angle[10] in Einsten's theory of gravity, it makes surrounding spacetime curved in scalar-tensor theories. Cosmic strings in various scalar-tensor gravity theories including dilaton gravity have been studied by many authors.[12]

In dilaton gravity theories, we consider a $U(1)$ gauge cosmic string formed in the early Universe and have cylindrically symmetric solutions of field equations far from the cosmic string. By comparing this vacuum solutions with the perturbative solutions obtained by Gregory and Santos,[12] we determine the spacetime metric around the string. Applying the formula (3.23) for rotation velocities to the solutions, we get a constant value of rotation velocities of stars in a galaxy seeded by the string, $v_{\text {rot. }}^{(s)} \propto G \eta^{2} \sim 10^{-6}$ which is to be given in Eq. (3.28), when we consider a cosmic string formed at the GUT scale of about $10^{16} \mathrm{GeV}$. This constant value obtained with a single string looks too small to explain the measured value[2] of rotation velocities in galactic halos. However, the value of rotation velocities around a bundle of a few hundred cosmic strings might be comparable to the measured value.[2] Even though such multi-string structure surrounded by the outer void region could be constructed by the possible force[13] of attraction between parallel strings or by the process of string accumulation,[14] we present a more concrete field-theoretical two-dilaton model, in illustration of the domain-wall structure[15] of strings. The flatness of spiral galaxy rotation curves might be due to the constant value of rotation velocities around the remnants of the multi-string, which has been possibly preserved because of the scaling property of the cosmic string network.[16] On the other hand, it can be also realized if we consider a cosmic string formed near the string-unification scale.[17] 
The rest of the paper is organized as follows. In Sec. 2, we derive equations of motion for $U(1)$ gauge fields $Z_{\mu}$ and a scalar field $\Phi^{0}$ coupled to dilaton gravity, and with appropriate ansatzes we reduce the equations to the cylindrically symmetric forms when the $U(1)$ symmetry is spontaneously broken. In Sec. 3, we find vacuum solutions far from the $Z$-string formed at the origin. We calculate the rotation velocities of objects circulating the cosmic string and discuss their relation to flat rotation curves in galaxies. In Sec. 4, we present a two-dilaton model for the flatness of rotation curves in the galactic halo region. The summary and discussion is given in Sec. 5 .

\section{Z-STRINGS IN DILATON GRAVITY THEORIES}

Since Jordan, Brans and Dicke[18] introduced a scalar field instead of $G^{-1}$ for the purpose of generalizing Einstein's theory of gravity to incorporate Mach's principle, various scalar-tensor theories of gravity have been studied. A fourdimensional dilaton gravity obtained as a low energy effective theory from fundamental strings also has the form of a scalar-tensor theory, with non-trivial couplings of the dilaton to matter and a possible dilaton potential. When a potential of the dilaton field, $e^{-2 \phi} V(\phi)$, is included, an action of dilaton gravity theories is given by

$$
S=\frac{1}{16 \pi G} \int d^{4} x \sqrt{-\tilde{g}} e^{-2 \phi}\left[\tilde{R}+4 \tilde{g}^{\mu \nu} \partial_{\mu} \phi \partial_{\nu} \phi-e^{-2 \phi} V(\phi)\right]+S_{m}
$$

with the action for dilaton-matter couplings, $S_{m}=\int d^{4} x \sqrt{-\tilde{g}} e^{2 a \phi} \mathcal{L}_{m}$.

Let us consider a theory of gauge fields and a scalar field with a broken $U(1)$ gauge symmetry in the dilaton gravity. By the conformal transformation

$$
\tilde{g}_{\mu \nu}=e^{2 \phi} g_{\mu \nu}
$$

from the above action we have the action of the theory in the socalled Einstein conformal frame.[19]

$$
S=\frac{1}{16 \pi G} \int d^{4} x \sqrt{-g}\left[R-2 g^{\mu \nu} \partial_{\mu} \phi \partial_{\nu} \phi-V(\phi)\right]+S_{m}\left[e^{2 \phi} g_{\alpha \beta}, Z_{\mu}, \Phi^{0}\right]
$$

where

$$
\begin{aligned}
S_{m}\left[e^{2 \phi} g_{\alpha \beta}, Z_{\mu}, \Phi^{0}\right]= & \int d^{4} x \sqrt{-g} e^{2(a+2) \phi} \mathcal{L}_{m}\left(e^{2 \phi} g_{\alpha \beta}, Z_{\mu}, \Phi^{0}\right) \\
= & -\int d^{4} x \sqrt{-g}\left[\frac{1}{4} e^{2 a \phi} Z^{\mu \nu} Z_{\mu \nu}+e^{2(a+1) \phi} g^{\mu \nu}\left(D_{\mu} \Phi^{0}\right)^{\dagger} D_{\nu} \Phi^{0}\right. \\
& \left.+e^{2(a+2) \phi} V_{\eta}\left(\Phi^{0}\right)\right] .
\end{aligned}
$$

Here $Z_{\mu}$ is a neutral gauge field and $Z_{\mu \nu}=\partial_{\mu} Z_{\nu}-\partial_{\nu} Z_{\mu}$ are components of its field strength with $Z^{\mu \nu}=g^{\mu \alpha} g^{\nu \beta} Z_{\alpha \beta}$. $\Phi^{0}$ is the scalar field, and its covariant derivative and potential are given by

$$
\begin{aligned}
D_{\mu} \Phi^{0} & =\left(\partial_{\mu}+i e Z_{\mu}\right) \Phi^{0} \\
V_{\eta}\left(\Phi^{0}\right) & =\lambda\left(\left|\Phi^{0}\right|^{2}-\eta^{2}\right)^{2} .
\end{aligned}
$$

Varying the action (2.3) with respect to the fields, we have following equations: The equation for the gauge fields $Z_{\mu}$ is

$$
\frac{1}{\sqrt{-g}} \partial_{\nu} e^{2 a \phi} \sqrt{-g} g^{\mu \alpha} g^{\nu \beta} Z_{\alpha \beta}=i e e^{2(a+1) \phi} g^{\mu \nu}\left[\Phi^{0^{\dagger}}\left(D_{\nu} \Phi^{0}\right)-\left(D_{\nu} \Phi^{0}\right)^{\dagger} \Phi^{0}\right]
$$

The equation for the scalar field $\Phi^{0}$ is given by

$$
\frac{1}{\sqrt{-g}} D_{\nu}^{\dagger} e^{2(a+1) \phi} \sqrt{-g} g^{\mu \nu}\left(D_{\nu} \Phi^{0}\right)^{\dagger}=2 \lambda e^{2(a+2) \phi}\left(\left|\Phi^{0}\right|^{2}-\eta^{2}\right) \Phi^{0^{\dagger}},
$$

and the equation for the dilaton field $\phi$

$$
\frac{1}{\sqrt{-g}} \partial_{\mu} \sqrt{-g} g^{\mu \nu} \partial_{\nu} \phi-\frac{1}{4} \frac{\partial V}{\partial \phi}=-\frac{4 \pi G}{\sqrt{-g}} \frac{\delta S_{m}}{\delta \phi}
$$


where

$$
\begin{aligned}
\frac{1}{\sqrt{-g}} \frac{\delta S_{m}}{\delta \phi} & =-\left[2(a+2) e^{2(a+2) \phi} \lambda\left(\left|\Phi^{0}\right|^{2}-\eta^{2}\right)^{2}\right. \\
& \left.+2(a+1) e^{2(a+1) \phi} g^{\alpha \beta}\left(D_{\alpha} \Phi^{0}\right)^{\dagger} D_{\beta} \Phi^{0}+\frac{a}{2} e^{2 a \phi} Z^{\alpha \beta} Z_{\alpha \beta}\right] .
\end{aligned}
$$

Einstein's field equations are given by

$$
G_{\mu \nu}=8 \pi G T_{\mu \nu}+2 \partial_{\mu} \phi \partial_{\nu} \phi-\frac{1}{2} g_{\mu \nu}\left\{2 g^{\alpha \beta} \partial_{\alpha} \phi \partial_{\beta} \phi+V(\phi)\right\},
$$

where the energy-momentum tensor

$$
\begin{aligned}
T_{\mu \nu} & \equiv-\frac{2}{\sqrt{-g}} \frac{\delta S_{m}}{\delta g^{\mu \nu}} \\
& =-\left[g_{\mu \nu}\left\{e^{2(a+2) \phi} \lambda\left(\left|\Phi^{0}\right|^{2}-\eta^{2}\right)^{2}+e^{2(a+1) \phi} g^{\alpha \beta}\left(D_{\alpha} \Phi^{0}\right)^{\dagger} D_{\beta} \Phi^{0}\right\}\right. \\
& \left.-2 e^{2(a+1) \phi}\left(D_{\mu} \Phi^{0}\right)^{\dagger} D_{\nu} \Phi^{0}+e^{2 a \phi}\left(\frac{1}{4} g_{\mu \nu} Z^{\alpha \beta} Z_{\alpha \beta}-g^{\alpha \beta} Z_{\alpha \mu} Z_{\beta \nu}\right)\right] .
\end{aligned}
$$

With the potential of the field $\Phi^{0}$ given by $V_{\eta}\left(\Phi^{0}\right)=\lambda\left(\left|\Phi^{0}\right|^{2}-\eta^{2}\right)^{2}$ in Eq. (2.5), we are interested in a string-like defect situated at the origin, and we thus take ansatzes for the scalar field and the gauge field as[20]

$$
\begin{array}{r}
\Phi^{0}=\eta f(\rho) e^{i n \varphi}, \\
Z_{\mu}=\frac{1}{e}[P(\rho)-n] \delta_{\mu}^{\varphi},
\end{array}
$$

and for the spacetime metric with cylindrical symmetry as[21]

$$
d s^{2}=g_{\mu \nu} d x^{\mu} d x^{\nu}=A(\rho)\left(-d t^{2}+d z^{2}\right)+d \rho^{2}+B(\rho) d \varphi^{2} .
$$

Defining new, dimensionless variable and quantities as

$$
\begin{aligned}
\sqrt{\lambda} \eta \rho & =r, \\
\frac{e^{2}}{\lambda} & =\alpha, \\
8 \pi G \eta^{2} & =\kappa, \\
\lambda \eta^{2} B & \rightarrow B,
\end{aligned}
$$

we have following equations from Eqs. (2.6)-(2.10), when $V(\phi)=\lambda \eta^{2} \mathcal{V}(\phi)$. The gauge field equation (2.6) reads

$$
\frac{1}{\Psi^{a} A B^{-\frac{1}{2}}}\left(\Psi^{a} A B^{-\frac{1}{2}} P^{\prime}\right)^{\prime}=2 \alpha \Psi f^{2} P .
$$

The scalar field equation (2.7) becomes

$$
\frac{1}{\Psi^{a+1} A B^{\frac{1}{2}}}\left(\Psi^{a+1} A B^{\frac{1}{2}} f^{\prime}\right)^{\prime}=2 \Psi\left(f^{2}-1\right) f+\frac{f P^{2}}{B} .
$$

The dilaton equation (2.8) becomes

$$
\frac{1}{A B^{\frac{1}{2}}}\left(A B^{\frac{1}{2}} \phi^{\prime}\right)^{\prime}-\frac{1}{4} \frac{\partial \mathcal{V}}{\partial \phi}=\kappa \Psi^{a}\left[\frac{a}{2} \frac{P^{\prime 2}}{\alpha B}+(a+2) \Psi^{2}\left(f^{2}-1\right)^{2}+(a+1) \Psi\left(f^{\prime 2}+\frac{f^{2} P^{2}}{B}\right)\right] .
$$

The $(\rho \rho)$-component of the Einstein equation (2.10) reads

$$
\frac{1}{2}\left(\frac{A^{\prime}}{A}\right)^{2}+\frac{A^{\prime}}{A} \frac{B^{\prime}}{B}+\mathcal{V}-2\left(\phi^{\prime}\right)^{2}=\kappa \Psi^{a}\left[2 \Psi f^{\prime 2}+\frac{P^{\prime 2}}{\alpha B}-2 \Psi^{2}\left(f^{2}-1\right)^{2}-2 \Psi \frac{f^{2} P^{2}}{B}\right] .
$$

The other components of the Einstein equation are reduced to the following two equations.

$$
\begin{aligned}
\frac{A^{\prime \prime}}{A}+\frac{1}{2} \frac{A^{\prime}}{A} \frac{B^{\prime}}{B}+\mathcal{V} & =\kappa \Psi^{a}\left[\frac{P^{\prime 2}}{\alpha B}-2 \Psi^{2}\left(f^{2}-1\right)^{2}\right], \\
\frac{B^{\prime \prime}}{B}+\frac{A^{\prime}}{A} \frac{B^{\prime}}{B}-\frac{1}{2}\left(\frac{B^{\prime}}{B}\right)^{2}+\mathcal{V} & =-\kappa \Psi^{a}\left[\frac{P^{\prime 2}}{\alpha B}+2 \Psi^{2}\left(f^{2}-1\right)^{2}+4 \Psi \frac{f^{2} P^{2}}{B}\right],
\end{aligned}
$$

where $\Psi \equiv e^{2 \phi}$ and $\frac{\partial}{\partial r} A=A^{\prime}, \ldots$ have been used for the simplicity. Above equations (2.15)-(2.20) are consistent with the result of Verbin et al.[22] when $\mathcal{V} \equiv 0$. 


\section{VACUUM SOLUTIONS AND CIRCULAR MOTION AROUND COSMIC STRING}

Let us consider the case $\mathcal{V}=0$ in this section. Far from the $Z$-string, $r>>1$, the vacuum solutions $\left(\left|\Phi^{0}\right| \simeq \eta\right)$ are given with the following boundary conditions.[20]

$$
f \simeq 1, P \simeq 0
$$

which give us, from Eqs. (2.15) and (2.16),

$$
\Psi^{a} A B^{\frac{-1}{2}} P^{\prime}=c_{p}, \quad \Psi^{a+1} A B^{\frac{1}{2}} f^{\prime}=c_{f}
$$

with constants $c_{p}$ and $c_{f}$. In case[20] of

$$
c_{p}=c_{f}=0
$$

we have

$$
f^{\prime} \simeq 0, \quad P^{\prime} \simeq 0
$$

which are consistent with Eq. (3.1). With these, Eq. (2.17) gives us

$$
A B^{\frac{1}{2}} \phi^{\prime}=c_{\phi}
$$

with a constant $c_{\phi}$.

The Einstein equations (2.18)-(2.20) read

$$
\begin{aligned}
\frac{1}{2}\left(\frac{A^{\prime}}{A}\right)^{2} & +\frac{A^{\prime}}{A} \frac{B^{\prime}}{B}-2\left(\phi^{\prime}\right)^{2}=0, \\
\frac{A^{\prime \prime}}{A} & +\frac{1}{2} \frac{A^{\prime}}{A} \frac{B^{\prime}}{B}=0, \\
\frac{B^{\prime \prime}}{B} & +\frac{A^{\prime}}{A} \frac{B^{\prime}}{B}-\frac{1}{2}\left(\frac{B^{\prime}}{B}\right)^{2}=0 .
\end{aligned}
$$

We can satisfy Eq. (3.7) with

$$
A^{\prime}=c_{a} B^{-\frac{1}{2}}
$$

and we have the equation

$$
B^{\prime}=c_{b} B^{\frac{1}{2}} A^{-1}
$$

with the relation between constants, $c_{\phi}^{2}=\frac{1}{4} c_{a}\left(c_{a}+2 c_{b}\right)$. With Eqs. (3.9)-(3.10), Eq. (3.8) reads

$$
\frac{B^{\prime \prime}}{B}+\left(\frac{c_{a}}{c_{b}}-\frac{1}{2}\right)\left(\frac{B^{\prime}}{B}\right)^{2}=0,
$$

whose solution is

$$
B=B_{i} r^{2-\epsilon}
$$

where

$$
\epsilon \equiv \frac{4 \frac{c_{a}}{c_{b}}}{1+2 \frac{c_{a}}{c_{b}}} .
$$

The solutions for another metric coefficient $A$ and the dilaton field $\phi$ are given by

$$
\begin{aligned}
A(r) & =A_{i} r^{\frac{\epsilon}{2}}, \\
\phi(r) & =\phi_{i}+c_{i}(\epsilon) \ln \frac{r}{r_{c}},
\end{aligned}
$$


with $A_{i}=B_{i}^{-\frac{1}{2}}\left(c_{a}+\frac{c_{b}}{2}\right)$ and $c_{i}(\epsilon)=\sqrt{\frac{1}{2} \epsilon\left(1-\frac{3}{8} \epsilon\right)}$. With these solutions the spacetime metric becomes

$$
d s^{2}=A_{i} r^{\frac{\epsilon}{2}}\left(-d t^{2}+d z^{2}\right)+d r^{2}+B_{i} r^{2-\epsilon} d \varphi^{2}
$$

To derive the formula for rotation velocities of objects circulating the string formed at $r=0$, we consider the following spacetime metric

$$
d s^{2}=g_{\mu \nu} d x^{\mu} d x^{\nu}=A(r)\left(-d t^{2}+d z^{2}\right)+d r^{2}+B(r) d \varphi^{2}
$$

which is obtained from the spacetime (2.13) with appropriate reparametrizations.[12] Using the definition

$$
\left(\frac{d t}{d \tau}, \frac{d r}{d \tau}, \frac{d z}{d \tau}, \frac{d \varphi}{d \tau}\right) \equiv(\dot{t}, \dot{r}, \dot{z}, \dot{\varphi})
$$

with the proper time $\tau$, from Eq. (3.16) with $\dot{z}=0$ we get the equation for the Lagrangian:

$$
\mathcal{L}(r, \dot{r}, \dot{\varphi}, \dot{t} ; \tau) \equiv-A(r) \dot{t}^{2}+\dot{r}^{2}+B(r) \dot{\varphi}^{2}=-1
$$

where it is used that $d s^{2}=-d \tau^{2}$ in our unit system. Since $\partial \mathcal{L} / \partial \varphi=0$ and $\partial \mathcal{L} / \partial t=0$, we have the constants of motion

$$
\frac{\partial \mathcal{L}}{\partial \dot{\varphi}} \equiv 2 B \dot{\varphi}=2 L, \quad \frac{\partial \mathcal{L}}{\partial \dot{t}} \equiv-2 A \dot{t}=-2 E .
$$

When $\dot{z}=0$, the geodesic equation in the spacetime (3.16), with the help of the above equations, reads

$$
\dot{r}^{2}+V_{\text {eff. }}(r)=0
$$

where

$$
V_{e f f .}(r)=1+\frac{L^{2}}{B(r)}-\frac{E^{2}}{A(r)}
$$

We require the following conditions for the objects to have circular motions:[23]

$$
\dot{r}=0, \frac{\partial V_{e f f .}}{\partial r}=0, \frac{\partial^{2} V_{e f f}}{\partial r^{2}}>0 .
$$

Following the same procedure as Refs. [23]-[24], we solve the above equations, express $\dot{\varphi}$ and $\dot{t}$ as functions of metric coefficients, and have the formula for the rotation velocity

$$
v_{\text {rot. }} \equiv \frac{B^{\frac{1}{2}}}{A^{\frac{1}{2}}} \frac{d \varphi}{d t}=\sqrt{\frac{A^{\prime} B}{A B^{\prime}}} .
$$

Applying the above equation to Eq. (3.15), we obtain the circular velocity of the objects around the string,

$$
v_{\text {rot. }}=\sqrt{\frac{c_{a}}{c_{b}}}=\sqrt{\frac{\epsilon}{4\left(1-\frac{\epsilon}{2}\right)}} .
$$

As we can see in Eq. (3.24), the velocities of rotating objects far away from $(r>>1)$ the cosmic string are non-zero and $r$-independent for $0 \leq \epsilon<2$. To discuss flat rotation curves in a galaxy whose formation was seeded by the cosmic string in the early Universe, we think of a situation where $0<\epsilon<2$ for $r_{c} \leq r \leq r_{h}$ and $\epsilon=0$ for $r_{h}<r$, with the radius of the galactic halo $r_{h}(\simeq 200-400 \mathrm{kpc})$.[25] We do not discuss the core region $\left(<r_{c}\right)$, since it might have very complex structure such as supermassive black hole.[26] For the outer void region $\left(>r_{h}\right)$ we take the value 0, instead of $\epsilon$, in Eqs. (3.12)-(3.15) and have a Minkowskian spacetime as follows.

$$
\begin{aligned}
B(r) & =B_{o} r^{2}, \\
A(r) & =A_{o}, \\
\phi(r) & =\phi_{o},
\end{aligned}
$$

where $B_{o}=B_{i} r_{h}^{-\epsilon}, A_{o}=A_{i} r_{h}^{\epsilon / 2}$, and $\phi_{o}=\phi_{i}+c_{i} \ln \left(r_{h} / r_{c}\right)$ are constants of integrations which are determined by the boundary conditions at $r=r_{h}$, and $B_{o}=B_{i} r_{h}^{-\epsilon}$ can be related with deficit angles.[10] 
Compared by the metric which was, to order $\eta^{4}$ in units of the Planck mass, determined by Gregory and Santos[12] and others,

$$
d s^{2}=\hat{r}^{(a+1)^{2}\left(\hat{\mu} \eta^{2} / 4\right)^{2}}\left(-d t^{2}+d \hat{r}^{2}+d z^{2}\right)+(1-\mu / 4 \pi) \hat{r}^{2-(a+1)^{2}\left(\hat{\mu} \eta^{2} / 4\right)^{2}} d \varphi^{2},
$$

the parameter $\epsilon$ can be given as

$$
\epsilon=\frac{(a+1)^{2} \frac{\hat{\mu}^{2} \eta^{4}}{8}}{1+(a+1)^{2} \frac{\hat{\mu}^{2} \eta^{4}}{32}} .
$$

With the string energy per unit length $\mu$ and $\mathcal{O}(1)$ quantity $\hat{\mu} \equiv \mu / 2 \pi \eta^{2}$, we have coordinate-transformed here as $\hat{r}^{1+(a+1)^{2} \hat{\mu}^{2} \eta^{4} / 32}=r$ in comparison with Eq. (3.15), and from Eq. (3.24) we get the velocity formula of objects rotating the single cosmic string

$$
v_{\text {rot. }}^{(s)} \simeq \frac{|a+1| \hat{\mu}}{4 \sqrt{2}} \eta^{2}
$$

which is useful in the region where the galactic halo exists, $r_{c} \leq r \leq r_{h}$.

If we consider a cosmic string formed at the GUT scale of $M_{G U T} \sim 10^{16} \mathrm{GeV}$, then we have a constant value of rotation velocities of stars in the string-seeded galaxy, $v_{\text {rot. }}^{(s)} \sim 10^{-6}$, from Eq. (3.28) with $a \neq-1$. This constant value by a single string looks too small to explain the measured value[2] $\left(\gtrsim 3 \times 10^{-4}\right)$ of rotation velocities in galactic halos. However, if a bundle of $N$ cosmic strings formed at $M_{G U T}$ seeded one galaxy, then the constant value of rotation velocities become much larger as $v_{\text {rot. }} \simeq N v_{\text {rot. }}^{(s)}$ and could be comparable to the measured value in the galactic halos, with $N \gtrsim 300$. The constant value of rotation velocities can remain from the era of the pregalaxies, with the help of the scaling solution for the cosmic string network,[16] and it can be related to the flatness of rotation curves in spiral galaxies. Such a domain-wall structure of strings explained below Eq. (3.24) can be realized also in a two-dilaton model, which is to be presented in the following section.

\section{TWO-DILATON MODEL}

In the last section we have suggested a very simple model for the explanation of flat rotation curves in the galactic halo, and now we present a more concrete, field-theoretical model in illustration of the domain-wall structure[15] of strings. Let us introduce another dilaton field $\chi$ in addition to the $\phi$ field with a $\phi$ - $\chi$ interation potential

$$
V_{\chi}(\phi, \chi)=\frac{\lambda_{\chi}}{2}\left[(\phi-\chi)\left(\phi-\phi_{0}\right)\right]^{2}
$$

The action in this model is given by

$$
S=\frac{1}{16 \pi G} \int d^{4} x \sqrt{-g}\left[R-2 g^{\mu \nu} \partial_{\mu} \phi \partial_{\nu} \phi-\frac{1}{2} e^{2 \delta \phi} g^{\mu \nu} \partial_{\mu} \chi \partial_{\nu} \chi-V_{\chi}(\phi, \chi)\right]+S_{m}
$$

where $S_{m}$ is as in Eq. (2.4).

Since $\frac{\partial V_{\chi}}{\partial \chi}=0, \frac{\partial V_{\chi}}{\partial \phi}=0$ and $V_{\chi}=0$ for $\phi=\chi$ or $\phi=\phi_{0}$, we have two different vacua $\phi=\chi$ or $\phi=\phi_{0}$, where we have following equations of motion for each dilaton field.

$$
\begin{gathered}
\frac{1}{\sqrt{-g}} \partial_{\mu} \sqrt{-g} g^{\mu \nu} \partial_{\nu} \chi+\lambda_{\chi}(\phi-\chi)\left(\phi-\phi_{0}\right)^{2}=0 \\
\frac{1}{\sqrt{-g}} \partial_{\mu} \sqrt{-g} g^{\mu \nu} \partial_{\nu} \phi-\frac{1}{4} \frac{\partial V}{\partial \phi}=-\frac{4 \pi G}{\sqrt{-g}} \frac{\delta S_{m}}{\delta \phi}+\frac{\delta}{4} e^{2 \delta \phi} g^{\mu \nu} \partial_{\mu} \chi \partial_{\nu} \chi
\end{gathered}
$$

The Einstein equation in this two-dilaton model is given by

$$
\begin{aligned}
G_{\mu \nu} & =8 \pi G T_{\mu \nu}+2 \partial_{\mu} \phi \partial_{\nu} \phi-\frac{1}{2} g_{\mu \nu}\left\{2 g^{\alpha \beta} \partial_{\alpha} \phi \partial_{\beta} \phi+V_{\chi}(\phi, \chi)\right\} \\
& +\frac{1}{2} e^{2 \delta \phi}\left\{\partial_{\mu} \chi \partial_{\nu} \chi-\frac{1}{2} g_{\mu \nu}\left(g^{\alpha \beta} \partial_{\alpha} \chi \partial_{\beta} \chi\right)\right\}
\end{aligned}
$$


which is same as Eq. (2.10), except its $(\rho \rho)$-component modified to

$$
\begin{aligned}
\frac{1}{2}\left(\frac{A^{\prime}}{A}\right)^{2}+\frac{A^{\prime}}{A} \frac{B^{\prime}}{B}+\mathcal{V}-2\left(\phi^{\prime}\right)^{2}-\frac{1}{2} e^{2 \delta \phi}\left(\chi^{\prime}\right)^{2} & =\kappa \Psi^{a}\left[2 \Psi f^{\prime 2}+\frac{P^{\prime 2}}{\alpha B}\right. \\
& \left.-2 \Psi^{2}\left(f^{2}-1\right)^{2}-2 \Psi \frac{f^{2} P^{2}}{B}\right] .
\end{aligned}
$$

With the same conditions as Eqs. (3.1)-(3.4) in the vacua in the spacetime (3.16), the $\chi$ field equation (4.3) reads

$$
\Psi^{\delta} A B^{\frac{1}{2}} \chi^{\prime}=c_{\chi}
$$

with a constant $c_{\chi}$, and the $\phi$ field equation is given by

$$
\left(A B^{\frac{1}{2}} \phi^{\prime}\right)^{\prime}=\frac{\delta}{4} \frac{c_{\chi}^{2}}{\Psi^{\delta} A B^{\frac{1}{2}}} .
$$

Therefore, Eq. (4.6) is reduced to

$$
\frac{1}{2}\left(\frac{A^{\prime}}{A}\right)^{2}+\frac{A^{\prime}}{A} \frac{B^{\prime}}{B}-2\left(\phi^{\prime}\right)^{2}=\frac{1}{2} e^{2 \delta \phi}\left(\chi^{\prime}\right)^{2},
$$

which is given instead of Eq. (3.6), while Eqs. (3.7) and (3.8) remain same as in the previous section. Eq. (3.7) can be satisfied with

$$
A^{\prime}=c_{a} B^{-\frac{1}{2}}
$$

as in the previous section, and we have the same relation as before,

$$
B^{\prime}=c_{b} B^{\frac{1}{2}} A^{-1} .
$$

In the simple case $\delta=0$, assuming

$$
B(r)=B_{i n} r^{2} r^{-\epsilon}
$$

for $r_{c} \leq r \leq r_{h}$ as Eq. (3.12), we have

$$
\begin{aligned}
A(r) & =A_{i n} r^{\frac{\epsilon}{2}} \\
\phi(r)-\phi_{i n} & =\chi(r)-\chi_{i n}=\frac{2}{\sqrt{5}} c_{i}(\epsilon) \ln \frac{r}{r_{c}},
\end{aligned}
$$

with $A_{\text {in }}=2 c_{a} B_{i n}^{-\frac{1}{2}} / \epsilon$ and $c_{i}(\epsilon)=\sqrt{\frac{1}{2} \epsilon\left(1-\frac{3}{8} \epsilon\right)}$. If we put $A_{i n}=A_{i}, B_{i n}=B_{i}$, and $\phi_{i n}+\frac{2}{\sqrt{5}} c_{i} \ln \left(r_{h} / r_{c}\right)=\phi_{o}$, then we have the same domain-wall structure of strings as in the previous section. However, $\phi=\chi$ in the galactic halo region $\left(<r_{h}\right)$ differently from the previous section, while $\phi=\phi_{o}$ in the outer void region $\left(>r_{h}\right)$.

\section{SUMMARY AND DISCUSSION}

In a spontaneously broken $U(1)$ gauge theory of gauge fields $\left(Z_{\mu}\right)$ and a scalar field, coupled to dilaton gravity, we have derived equations of motion for each fields with appropriate ansatzes in a cylindrically symmetric spacetime. With the solutions obtained far away from the $Z$-string formed at the origin, we have calculated the rotation velocities of the objects circulating the cosmic string and suggested their relation to flat rotation curves in the string-seeded galaxy. If the cosmic string formed at the typical GUT energy scale of $10^{16} \mathrm{GeV}$ grew into the galaxy, then we have a constant value of rotation velocity around it, $v_{\text {rot. }}^{(s)} \propto \eta^{2}\left(\sim 10^{-6}\right)$ in Eq. (3.28), which seems too small. However, the possible force[13] of attraction between parallel strings (or the process of string accumulation[14]) could make a bundle of multi-strings from the cosmic strings, and the constant value of rotation velocities around the bundle become much larger[27] and could be comparable to the measured one[2]. The flatness of spiral galaxy rotation curves might be their remaining property, which has been preserved from the era when the pregalaxies were made of the cosmic strings. This is possible due to the scaling[16] property for the cosmic string network, but for a more concrete conclusion we need numerical studies. 
On the other hand, if the cosmic sting were formed when the $U(1)$ symmetry was broken at extremely high energy near the string-unification scale $\left(\sim 5 \times 10^{17} \mathrm{GeV}\right),[17]$ we could have the constant value of rotation velocity comparable to the measured one[2] in the galaxy, which is including the remnants of the single string. However, for the explanation to be valid for flat rotation curves we should consider scalar-tensor gravity theories with $a \neq-1$ as we can see in Eq. (3.28). A simple model for the string-seeded galactic halo with two-dilaton fields has been constructed in the last section, with the case $\delta=0$ considered only. Two-dilaton fields without their potential terms such as Eq. (4.1) have been considered elsewhere, for example, in a two-dilaton model of electroweak interactions. [28] The case[29] $\delta \neq 0$ can be similarly studied, but the solution of the dilaton field $\phi(r)$ for $r<r_{h}$ seems not to be expressed in elementary functions. Our consideration in this paper has been restricted to the static spacetime with cylindrical symmetry, and it seems interesting to generalize ours to the cylindrical spacetime with rotation[30].

\section{Acknowledgments}

The author would like to thank his family for their helps. This work was supported by the Soongsil University Research Fund.

[1] T. W. B. Kibble, J. Phys. A9, 1387 (1976); Phys. Rep. 67, 183 (1980); Y. B. Zel'dovich, Mon. Not. Roy. Astron. Soc. 192, 663 (1980); A. Vilenkin, Phys. Rev. Lett. 46, 1169, 1496(E) (1981); Phys. Rev. D24, 2082 (1981); D. P. Bennett and S. H. Rhie, Phys. Rev. Lett. 65, 1709 (1990); Astrophys. J. Lett. 406, L7 (1993); M. Yamaguchi, Phys. Rev. D65, 063518 (2002).

[2] V. C. Rubin, W. K. Ford, and N. Thonnard, Astrophy. J. 238, 471 (1980); M. Persic and P. Salucci, Astrophys. J. Suppl. Ser. 99, 501 (1995); Y. Sofue, astro-ph/9906224.

[3] M. Barriola and A. Vilenkin, Phys. Rev. Lett. 63, 341 (1989).

[4] U. Nucamendi, M. Salgado, and D. Sudarsky, Phys. Rev. Lett. 84, 3037 (2000); Phys. Rev. D63, 125016 (2001); A. Banerjee, A. Beesham, S. Chatterjee, and A. A. Sen, gr-qc/9710002.

[5] D. Harari and C. Loustó, Phys. Rev. D42, 2626 (1990).

[6] L.A. Boyle, R.R. Caldwell and M. Kamionkowski, Phys. Lett. B545, 17 (2002); S. Kasuya, Phys. Lett. B515, 121 (2001); X. Li, J. Hao, and D. Liu, Class. Quantum Grav. 19, 6049 (2002).

[7] V. Faraoni, Phys. Rev. D62, 023504 (2000), and references therein; B. J. Lee and T. H. Lee, Int. J. Mod. Phys. A17, 555 (2002).

[8] T. H. Lee and B. J. Lee, Phys. Rev. D69, 127502 (2004).

[9] O. Dando and R. Gregory, gr-qc/9709029; A. Barros and C. Romero, Phys. Lett. A245, 31 (1998); C. Alcock et al., Astrophys. J. 449, 28 (1995); P. T. de Zeeuw, N. W. Evans, and M. Schwarzschild, Mon. Not. Roy. Astron. Soc. 280, 903 (1996); M. Milgrom, Astrophys. J. 270, 365 (1983); G. Dvali et al., Mod. Phys. Lett. A16, 513 (2001); M. E. Soussa and P. Woodard, Class. Quant. Grav. 20, 2737 (2003); T. H. Lee and B. H. J. McKellar, Phys. Rev. D67, 103007 (2003).

[10] A. Vilenkin, Phys. Rev. D23, 852 (1981); J. R. Gott III, Astrophys. J. 288, 422 (1985); W. Hiscock, Phys. Rev. D31, 3288 (1985); B. Linet, Gen. Relativ. Gravit. 17, 1109 (1985); D. Garfinkle, Phys. Rev. D32, 1323 (1985); D. Harari and P. Sikivie, Phys. Rev. D37, 3438 (1988); A. Vilenkin and E. P. S. Shellard, Cosmic Strings and Other Topological Defects (Cambridge Univ. Press, 1994); A. A. Sen and N. Banerjee, Phys. Rev. D62, 047302 (2000), and references therein.

[11] L. Yulin, Superconducting Cosmic String Evolution of Quasars, ICTP-preprint IC-88-279.

[12] R. Gregory and C. Santos, Phys. Rev. D56, 1194 (1997); C. Gundlach and M. Ortiz, Phys. Rev. D42, 2521 (1990); M. E. X. Guimaraes, Class. Quantum Grav. 14, 435 (1997).

[13] A. N. Aliev, M. Hortacsu, and N. Ozdemir, Class. Quantum Grav. 14, 3215 (1997); A. N. Aliev and N. Ozdemir, Class. Quantum Grav. 15, 3937 (1998).

[14] B. Gradwohl, Phys. Rev. D44, 1685 (1991).

[15] G. Dvali and G. Senjanovic, Phys. Rev. Lett. 72, 9 (1994); M. Nagasawa and M. Kawasaki, Phys. Rev. D50, 4821 (1994).

[16] E. J. Copeland, T. W. B. Kibble, and D. A. Steer, Phys. Rev. 58, 043508 (1998); J. A. Peacock, Cosmological Physics (Cambridge University Press, 1999).

[17] M. Green, J. Schwarz, and E. Witten, Superstring Theory, (Cambridge University Press, 1987); B. C. Allanach and S. F. King, Nucl. Phys. B473, 3 (1996); J. Rizos and K. Tamvakis, Phys. Lett., B414, 277 (1997), and references therein.

[18] P. Jordan, Z. Phys. 157, 112 (1959); C. Brans and R. H. Dicke, Phys. Rev. 124, 925 (1961).

[19] T. Damour, G. W. Gibbons, and C. Gundlach, Phys. Rev. Lett. 64, 123 (1990).

[20] B. Linet, Phys. Lett. A124, 240 (1987).

[21] K. S. Thorne, Phys. Rev. 138, 251 (1965).

[22] Y. Verbin, S. Madsen, A. L. Larsen, and M. Christensen, Phys. Rev. D65, 063503, (2002).

[23] T. Matos, F. S. Guzmán, and L. A. Ureña-López, in Exact Solutions and Scalar Fields in Gravity: Recent Developments, eds. A. Macias, J. L. Cervantes-Cota, and C. Lammerzahl (Plenum, 2001), pp. 166-184; T. Matos, F. S. Guzmán, and D. 
Núñez, Phys. Rev. D62, 061301(R) (2000); F. S. Guzmán and T. Matos, Class. Quantum Grav. 17, L9 (2000); Rev. Mex. Astron. Astrofis. 37, 63 (2001).

[24] S. Chandrasekhar, Mathematical Theory of Black Holes, (Oxford Science Publications, 1983).

[25] A. S. Kulessa and D. Lynden-Bell, Mon. Not. R. Astron. Soc. 255, 105 (1992); C. S. Kochanek, Astrophys. J. 457, 228 (1996); D. Zaritzky, R. Smith, C. S. Frenk, and S. D. M. White, astro-ph/9611199.

[26] A. M. Ghez, B. L. Klein, M. Morris, and E. E. Becklin, Astrophys. J. 509, 678 (1998).

[27] S. R. Nowling, Defect Induced Structure Formation in the Early Universe, unpublished.

[28] D. Kimberly and J. Magueijo, Phys. Lett. B584, 8 (2004).

[29] T. Ortin, Phys. Rev. D47, 3136 (1993); E. J. Copeland, J. E. Lidsey, and D. Wands, Phys. Lett. B443, 97 (1998).

[30] P. R. C. T. Pereira, N. O. Santos, and A. Z. Wang, Class. Quantum Grav. 13, 1641 (1996), L. Herrera, F. M. Paiva, and N. O. Santos, Class. Quantum Grav. 17, 1549 (2000), and references therein. 